\title{
Anti-moulting Activity in Brazilian Melia azedarach
}

\section{Marise MO Cabral /+ , Eloi S Garcia, Heinz Rembold* Salvatore G De Simone**, Alphonse Kelecom**}

\author{
Departamento de Bioquímica e Biologia Molecular, Instituto Oswaldo Cruz, Av. Brasil 4365, 21045-900 \\ Rio de Janeiro, RJ, Brasil *Max-Planck Institut fur Biochemie, Martinsried bei Munchen, D-8033, \\ Deutchland **Instituto de Biologia, Universidade Federal Fluminense, Caixa Postal 100.436, \\ 24001-970 Niterói, RJ, Brasil
}

Key words : Melia azedarach L. - seeds - anti-moulting activity

\begin{abstract}
Efficient control of Chagas disease depends heavily on an efficient control of its vectors, namely triatomid infested haematophagous insects, such as Rhodnius prolixus and some other related ones (ES Garcia, P Azambuja 1991 Parasitol Today 7: 240-244). Synthetic insecticides are commonly used for that purpose although they offer a series of disadvantages as they are both highly toxic and not specific. In addition, pest resistence to many pesticides has grown rapidly in recent years (H Schmutterer 1990 Annu Rev Entomol 35: 271-297). Nowadays, new chemicals used for insect control must be biodegradable. Such chemicals are expected to be found among natural products from higher plants, that are known to be rich sources of repellent and/or toxic secondary metabolites (B Subrahmanyam 1990 Proc Ind Acad Sci 99: 277-288). Azadirachtin-A, for example, is endowed of remarkable phagoinhibitor and anti-moulting activities on $R$. prolixus and might therefore be a serious candidate for Chagas disease control (ES Garcia et al. 1984 Z Naturforsch 39c: 1155-1158). However, it seems that production of azadirachtin-A may suffer strong biogeographic dependance since it is found in the Kenyan Melia azedarach (ED Morgan, MD Thornton 1973 Phytochem 12: 391-392), but not in crude extracts of the Paraguayan plant that are devoid of anti-moulting activity (AR Arias, GS Hirschmann 1988 Fitoterapia 59: 148-149).
\end{abstract}

This research note describes the anti-moulting activity observed in the methanolic crude ex-

\footnotetext{
This work was supported by CNPq, DAAD and Volkswagen Fundation grants.

${ }^{+} \mathrm{CNPq}$ and FAPERJ fellowship

Received 14 May 1995

Accepted 1 September 1995
}

tract and in several chromatographic fractions obtained from the seeds of M. azedarach collected in the neighbourhood of Niterói (State of Rio de Janeiro, Brazil). The bioassays were carried out as follows. Fourth instar nymphs of $R$. prolixus were used. Test material was dissolved in EtOHsalina (1:4) and aliquots were added to blood in order to obtain the desired final concentrations, between 10 and $100 \mu \mathrm{g} / \mathrm{ml}$. At higher concentrations, the crude extract showed phagoinhibition that precluded the study of the anti-moulting activity. Test blood was placed in special designed feeders, and the insects were allowed to feed (ES Garcia, H Rembold 1984 J Insect Physiol 30: 939941). After this, the insects were weighted, incubated at $28^{\circ} \mathrm{C}$ and observed every two days over a one month period. Only fully fed insects were used, partially fed ones were discarded. Death and ecdysis were counted.

Seeds of M. azedarach were extracted exhaustively with $\mathrm{MeOH}$ (yield 12\%). Filtration and evaporation of the solvent under reduced pressure furnished the crude extract that showed, depending on the concentration (see above), phagoinhibitor or anti-moulting activity. Only the latter was considered in the present study. All purification steps were bioassay guided. Thus, fractionation of the crude extract, by solvent-solvent partition between 5\% aq $\mathrm{MeOH}$ and hexane, then EtOAc, furnished an EtOAc fraction (A) that contained almost all the activity. Purification of a $1 \mathrm{~g}$ aliquot of $\mathrm{A}$, by repetitive chromatographic processes using silica gel and mixtures of $\mathrm{CHCl}_{3}-\mathrm{MeOH}$, furnished fraction $\mathrm{B}$ (21 $\mathrm{mg}$ ) that inhibited $100 \%$ moulting of $R$. prolixus at $25 \mu \mathrm{g} / \mathrm{ml}$ blood (Fig.). HPLC analysis, on a RP-18 column (eluent : $\mathrm{MeOH}-\mathrm{H}_{2} \mathrm{O}$ 45:55), indicated the presence, in the crude extract, of traces of a constituent whose retention time was identical to that of an authentic sample of azadirachtinA. In fraction $\mathrm{B}$, the concentration of this con- 
stituent raised to $13 \%$. The remaining fractions of this purification step contained, among the major constituents, classical phytosterols (cholesterol, campesterol and stigmasterol), four lignanes and one triterpene, all of which devoid of anti-moulting activity. Structure determination of these compounds will be described elsewhere.

The observation of strong biological activity in the methanolic extract of seeds of the Brazilian $M$. azedarach makes of this plant a potential tool of major interest in the control of Chagas disease in the same way as Azadirachta indica has been claimed to present an enormous potential for pest control (H Schmutterer 1990 Annu Rev Entomol 35: 271-297).

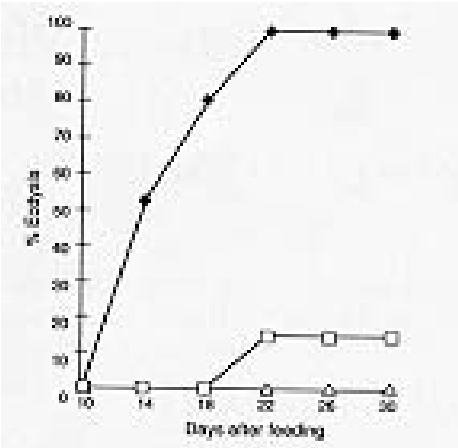

Effects of fraction B on ecdysis in fourth-instar nymphs of Rhodnius prolixus. Controls ( $)$, fraction B at concentrations of $10 \mu \mathrm{g} / \mathrm{ml}(\square)$ and $25 \mu \mathrm{g} / \mathrm{ml}(\Delta)$. Percentage of moults was calculated 30 days after feeding. Groups of 15-20 nymphs. 